\title{
SCHRÖDINGER OPERATORS WITH FEW BOUND STATES
}

\author{
DAVID DAMANIK, ROWAN KILLIP, AND BARRY SIMON
}

\begin{abstract}
We show that whole-line Schrödinger operators with finitely many bound states have no embedded singular spectrum. In contradistinction, we show that embedded singular spectrum is possible even when the bound states approach the essential spectrum exponentially fast.

We also prove the following result for one- and two-dimensional Schrödinger operators, $H$, with bounded positive ground states: Given a potential $V$, if both $H \pm V$ are bounded from below by the ground-state energy of $H$, then $V \equiv 0$.
\end{abstract}

\section{INTRODUCTION}

This paper has its roots in the following result of Killip and Simon [14: A discrete whole-line Schrödinger operator has spectrum $[-2,2]$ if and only if the potential vanishes identically.

To be more precise, given a potential $V: \mathbb{Z} \rightarrow \mathbb{R}$, we define the Schrödinger operator

$$
\left[h_{V} \phi\right](n)=\phi(n+1)+\phi(n-1)+V(n) \phi(n)
$$

on $\ell^{2}(\mathbb{Z})$. The theorem mentioned above, [14, Theorem 8], says that $\sigma\left(h_{V}\right) \subseteq[-2,2]$ implies $V \equiv 0$. A simple variational proof of this theorem was given in 4, where the result was also extended to two dimensions; it does not hold in three or more dimensions, nor on the half-line.

It was also shown in 4 that, for bounded potentials, the essential spectrum of $h_{V}$ is contained in $[-2,2]$ if and only if $V \rightarrow 0$. This shows that $\sigma_{\text {ess }}\left(h_{V}\right)=[-2,2]$ in this case.

Damanik and Killip, 6], investigated half-line Schrödinger operators with spectrum contained in $[-2,2]$. By a half-line Schrödinger operator we mean an operator of the form (1), acting on $\ell^{2}\left(\mathbb{Z}^{+}\right)$, with $\phi(-1)=0$. We will denote this operator by $h_{V}^{+}$. It was shown that if $\sigma\left(h_{V}^{+}\right)=[-2,2]$, then $h_{V}^{+}$has purely absolutely continuous spectrum. Using the methods developed to prove this result, it was also shown that half-line Schrödinger operators with finitely many bound states (i.e., eigenvalues lying outside $[-2,2]$ ) have purely absolutely continuous spectrum on $[-2,2]$. See also Simon 23 .

Several people have asked, in private, whether one can deduce absence of embedded singular spectrum from

$$
\sum_{n} \sqrt{\left|E_{n}\right|-2}<\infty .
$$

Date: February 7, 2008.

D. D. was supported in part by NSF grant DMS-0227289.

R. K. was supported in part by NSF grant DMS-0401277.

B. S. was supported in part by NSF grant DMS-0140592. 
(This condition often arises as a natural borderline condition for regular behavior of the spectral measure near the points -2 and 2 ; see for example [17, 25]. It is equivalent to the convergence of the Blaschke product associated with the eigenvalues after the cut plane is mapped to the unit disk by the inverse of $z \mapsto z+z^{-1}$.)

In fact, embedded singular spectrum can occur even when the bound states approach \pm 2 exponentially fast. This is our first result.

Theorem 1. There is a discrete half-line Schrödinger operator with zero as an eigenvalue, whose bound states obey $\left|E_{n}\right|-2 \leq c^{n}$ for a suitable $c<1$.

Remark. As we will show, $\left|E_{n}\right|-2$ also admits an exponential lower bound. We do not know whether the existence of embedded singular spectrum places a lower bound on the rate at which the eigenvalues can approach $[-2,2]$. On the other hand, recent work of Damanik and Remling, 8, shows that finiteness of the $p$-th eigenvalue moment implies that the embedded singular spectrum must be supported on a set of Hausdorff dimension $4 p$.

The Damanik-Killip paper, [6], discussed earlier also considered half-line continuum Schrödinger operators with a Dirichlet boundary condition. That is, given a potential $V$ we define an operator on $L^{2}\left(\mathbb{R}^{+}\right)$by

$$
\left[H_{V}^{+} \phi\right](x)=-\phi^{\prime \prime}(x)+V(x) \phi(x)
$$

with $\phi(0)=0$. In this case it was proved that if $V$ belongs to the space $\ell^{\infty}\left(L^{2}\right)$ of uniformly locally square-integrable functions and both $H_{V}^{+}$and $H_{-V}^{+}$have only finitely many bound states, then both have purely absolutely continuous spectrum on $[0, \infty)$. This is the continuum analogue of the Damanik-Killip theorem discussed above, as we will now explain: the unitary map $\phi(n) \mapsto(-1)^{n} \phi(n)$ conjugates $h_{-V}$ to $-h_{V}$; consequently, $h_{V}$ has finitely many bound states if and only if both $2-h_{ \pm V}$ have only finitely many bound states below zero.

It is natural to ask what one may say for whole-line Schrödinger operators with finitely many bound states. (We write $H_{V}$ for the operator on $L^{2}(\mathbb{R})$ defined through (2).) The analogous results hold:

Theorem 2. If the operator $h_{V}$ has only finitely many bound states, then it has purely absolutely continuous spectrum of multiplicity 2 on $[-2,2]$.

Theorem 3. Suppose $V \in \ell^{\infty}\left(L^{2}\right)$. If both $H_{V}$ and $H_{-V}$ have only finitely many eigenvalues below energy zero, then both operators have purely absolutely continuous spectrum of multiplicity 2 on the interval $[0, \infty)$.

Recall that if the operators $h_{ \pm V}$ are required to have no bound states above 2 , then $V$ must be identically 0 - this is the result of Killip-Simon. We will prove the continuum analogue of this result by revisiting the variational approach of 4 . Moreover, the refinements we introduce permit us to treat more general background potentials.

We begin with some notation. Expanding our previous usage, we let $H_{V}$ denote the Schrödinger operator with potential $V$ in $L^{2}\left(\mathbb{R}^{d}\right)$ for any dimension $d$. By a ground state for $H_{V}$, we mean a (distributional) solution of $-\nabla^{2} \psi+V \psi=E \psi$ where $E$ is the ground-state energy, that is, the infimum of the spectrum. We use the analogous terminology in the discrete case; however, here we define the ground-state energy to be the supremum of the spectrum. 
Theorem 4. Let $d=1$ or 2. Suppose that $Q, V$ are functions on $\mathbb{Z}^{d}$ such that the operator $h_{Q}$ has a bounded positive ground state and both $h_{Q \pm V}$ are bounded above by the ground state energy of $h_{Q}$. Then $V \equiv 0$.

Theorem 5. Let $d=1$ or 2 . Suppose that $Q \in L_{\mathrm{loc}}^{2}\left(\mathbb{R}^{d}\right)$ and the operator $H_{Q}$ has a bounded positive ground state. If $V \in L_{\mathrm{loc}}^{2}\left(\mathbb{R}^{d}\right)$ and both $H_{Q \pm V}$ are bounded below by the ground state energy of $H_{Q}$, then $V \equiv 0$.

As noted already in [4], these theorems fail for $d \geq 3$ even when $Q \equiv 0$. This is an immediate consequence of the Cwikel-Lieb-Rosenblum inequality. However, the presence of absolutely continuous spectrum may still be deduced in some situations as a recent result of Safronov shows [21].

The existence of a positive ground state holds under fairly general conditions; see Simon [24]. Moreover, it is not hard to see that, for periodic potentials $Q$, the ground state energy corresponds to zero quasi-momentum (this can also be derived directly from more general results of Agmon [1]) and hence the operator $H_{Q}$ has a bounded positive ground state. Thus, as an application of Theorem [5 we may deduce

Corollary 1. Let $d=1$ or 2 and suppose that $Q \in L_{\mathrm{loc}}^{2}\left(\mathbb{R}^{d}\right)$ is periodic. If $V \in$ $L_{\text {loc }}^{2}\left(\mathbb{R}^{d}\right)$ and both $H_{Q \pm V}$ are bounded below by the ground state energy of $H_{Q}$, then $V \equiv 0$.

The particular case $d=1$ and $Q \equiv 0$ yields the continuum analogue of the Killip-Simon theorem mentioned at the beginning of the introduction. The discrete analogue of the corollary also holds and therefore extends the original Killip-Simon result to the case of periodic background.

Y. Pinchover has explained to us that Theorem 5 represents a statement about the criticality of $H_{Q}$ (cf. [18, 19]). He also pointed out that through these techniques, Corollary 1$]$ can be derived from Theorem 2 of [20].

We will use Dirac notation for the inner product of the underlying Hilbert space $\mathcal{H}$. In particular, if $H$ is a self-adjoint operator in $\mathcal{H}$ and $\phi, \psi$ belong to the form domain of $H$, then we write the quadratic form associated with $H$ as $\langle\phi|H| \psi\rangle$.

The organization is as follows. In Section 2 we discuss an example with an embedded eigenvalue and exponential bound state decay and, in particular, prove Theorem 11 Theorems 2 and 3 are proven in Section 3 Finally, we study perturbations of Schrödinger operators with positive ground states in Section 4 and obtain Theorems 4 and 5

Acknowledgment. It is our pleasure to thank Y. Pinchover and A. V. Sobolev for useful comments.

\section{Embedded Eigenvalue and Exponential Bound State Decay}

The following result contains Theorem 1 and also provides an exponential lower bound.

Theorem 6. There is a discrete half-line Schrödinger operator that has zero as an eigenvalue and its eigenvalues $\left\{E_{n}\right\}$ outside $[-2,2]$ obey $b^{n} \lesssim\left|E_{n}\right|-2 \lesssim c^{n}$ for suitable $b \leq c<1$.

Remark. For functions $f, g$, we write $f \lesssim g$ if $f / g$ is bounded. 
We will revisit an example of Wigner-von Neumann type discussed in 6]. It is roughly of the form $V(n) \sim \lambda(-1)^{n} n^{-1}$. It follows from 4 that $\lambda$ must be of magnitude greater than one in order for the operator to have infinitely many eigenvalues outside $[-2,2]$.

By Weyl's theorem, the spectrum outside $[-2,2]$ consists of eigenvalues, $E_{n}(V)$, that can accumulate only at \pm 2 . We choose an ordering such that $\left|E_{1}(V)\right| \geq$ $\left|E_{2}(V)\right| \geq \cdots$.

Proof of Theorem [6] Fix $\alpha>1 / 2$ and define $\psi: \mathbb{Z}^{+} \rightarrow \mathbb{R}$ as follows: the absolute value is given by $|\psi(n)|=(n+1)^{-\alpha}$ and the sign depends on the value of $n \bmod$ 4 with the pattern,,,,$++-- \ldots$. Notice that $\psi$ is square-summable and so a zero-energy eigenfunction for the operator $h_{V}$ with potential given by

$$
V(n)=-\frac{\psi(n+1)+\psi(n-1)}{\psi(n)}
$$

for $n \geq 1$ and $V(0)=-\psi(1) / \psi(0)$. Clearly,

$$
V(n)=-2 \alpha(-1)^{n} n^{-1}+O\left(n^{-2}\right)
$$

We now turn to the main part of the proof: controlling the bound states of $h_{V}$. Both inequalities rely on results relating operators with sign indefinite potentials to those with sign-definite potentials. We begin with upper bounds.

It was shown in [5] (see, in particular, Eq. (4.18)) that if $V^{ \pm}$are defined by

$$
V^{ \pm}(n)= \pm 2\left[F(n)^{2}+F(n+1)^{2}\right] \quad \text { with } \quad F(n)=-\sum_{j=n}^{\infty} V(j),
$$

then

$$
2-h_{V} \geq \frac{1}{2}\left(2-h_{V^{+}}\right) \quad \text { and } \quad 2+h_{V} \geq \frac{1}{2}\left(2+h_{V^{-}}\right) .
$$

Thus it suffices to bound the eigenvalues for $h_{V^{ \pm}}$. To do this we employ the Jacobi matrix analogue of the Bargmann bound (see [11, Theorem A.1]):

$$
\#\left\{\left|E_{n}\left(V^{ \pm}\right)\right| \geq \lambda+2\right\} \lesssim \sum(n+1)|| V^{ \pm}(n)|-\lambda|_{+} .
$$

where $|x|_{+}=\max \{x, 0\}$. In our case, $\left|V^{ \pm}(n)\right| \lesssim(n+1)^{-2}$ and so $\#\left\{\left|E_{n}\left(V^{ \pm}\right)\right| \geq\right.$ $\lambda+2\} \lesssim \log (\lambda)$, or equivalently, there exists $0<c<1$ so that $\left|E_{n}\left(V^{ \pm}\right)\right|-2 \lesssim c^{n}$.

We now turn to a proof of the lower bound for $\left|E_{n}(V)\right|$. We will employ some ideas and results of 4 . From Eq. (1.7) of that paper we see that in order to prove $\left|E_{n}(V)\right|-2 \gtrsim b^{n}$ for some $0<b<1$, it suffices to find trial functions $\varphi_{n}$, whose supports are disjoint, such that

$$
\left\langle\varphi_{n}\left|h_{\tilde{V}}-2\right| \varphi_{n}\right\rangle \gtrsim b^{n}
$$

where $\tilde{V}(n)=\frac{1}{4} V(n)^{2}$. Note that from (3),

$$
\tilde{V}(n)=\alpha^{2} n^{-2}+O\left(n^{-3}\right) .
$$

For convenience, we pick $\alpha \geq \sqrt{7}$ (the construction below can be modified to accommodate any $\alpha>1 / 2)$. Let $m_{n}=8^{n}$. The trial function $\varphi_{n}$ is then the function which is 1 at $m_{n}$, has constant slope on the intervals $\left[\frac{m_{n}}{4}-1, m_{n}\right]$ and $\left[m_{n}, \frac{3 m_{n}}{2}+1\right]$, and vanishes outside the interval $\left[\frac{m_{n}}{4}-1, \frac{3 m_{n}}{2}+1\right]$. Mimicking the arguments from the proof of [4, Theorem 5.5], we see that

$$
\left\langle\varphi_{n}\left|h_{\tilde{V}}-2\right| \varphi_{n}\right\rangle \geq \frac{1}{m_{n}}
$$


and hence (5) holds with $b=1 / 8$. This concludes the proof.

Basically, the argument that the eigenvalues have geometrically fast approach to \pm 2 comes from the quadratic mapping (44) and the fact that for supercritical $r^{-2}$ potentials, the approach is geometric. This was shown in the continuum case by Kirsch and Simon [15] with explicit constants. It should be possible to compute $\lim \left(\left|E_{n}\right|-2\right)^{1 / n}$ in the discrete setting along similar lines. Bounds of this form can be used in the study of the Efimov effect; see Tamura [27, for example. The fact that there are infinitely many bound states for coupling above a critical value was shown in the discrete case by Năman [16].

\section{Whole-Line Operators With Finitely Many Bound States}

The purpose of this section is to prove Theorems 2 and 3

By restricting a whole-line operator $h_{V}$ to $\ell^{2}\left(\mathbb{Z}^{ \pm}\right)$we obtain two half-line operators, which we denote by $h_{V}^{ \pm}$. (Here $\mathbb{Z}^{+}=\{0,1,2, \ldots\}$ and $\mathbb{Z}^{-}=\{-1,-2,-3, \ldots\}$.)

If $h_{V}$ has finitely many bound states, then so do both $h_{V}^{ \pm}$because their direct sum is a finite-rank perturbation of $h_{V}$. Thus, it follows from [6] that both $h_{V}^{ \pm}$have purely absolutely continuous spectrum (essentially supported) on $[-2,2]$. Using the finite-rank perturbation property again, it follows that $h_{V}$ has absolutely continuous spectrum of multiplicity two (essentially supported) on $[-2,2]$.

Singular spectrum, or its absence, is not stable under finite-rank perturbations. We will revisit the half-line proof from [6], which proceeded through controlling the behavior of solutions and then applying the Jitomirskaya-Last version, 13, of the Gilbert-Pearson theory of subordinacy, 9, 10. The whole-line extension of the Jitomirskaya-Last result that we need can be found in [7]. We begin by recalling the necessary results from [6, 7].

Let us write $\psi_{\theta}$ for the solution of

$$
\psi(n+1)+\psi(n-1)+V(n) \psi(n)=E \psi(n)
$$

that obeys the initial condition

$$
\psi_{\theta}(-1)=\sin (\theta), \quad \psi_{\theta}(0)=\cos (\theta) .
$$

Proposition 3.1. Suppose that the operator $h_{V}^{+}$has only finitely many eigenvalues outside $[-2,2]$. Then for any energy $0<|E|<2$ and any $\eta>1 / \sqrt{2}$,

$$
n^{-\eta} \lesssim\left|\psi_{\theta}(n)\right|^{2}+\left|\psi_{\theta}(n+1)\right|^{2} \lesssim n^{\eta}
$$

for all $\theta$ and $n>0$. The implicit constants depend on $E$ and $\eta$, but not $\theta$. For $E=0$, (7) holds with $\eta=1$.

Proof. This follows from Corollary 4.6 and Proposition 5.2 of [6]. While the statement given there does not describe the dependencies of the constants, they can be deduced readily from the proof.

Proposition 3.2. Suppose that the operators $h_{V}^{ \pm}$have only finitely many eigenvalues outside $[-2,2]$. Then the set of energies in $(-2,2)$ for which (6) has unbounded solutions is of Hausdorff dimension zero.

Proof. As an unbounded solution of (6) must be unbounded on one side of the origin, we may apply the half-line results of [6], specifically, Corollary 4.6 and Proposition 5.5. 
The following result can be found in [7]:

Proposition 3.3. Suppose that for some $\eta<1$ and each energy $E$ in a bounded set $A$,

$$
n^{-\eta} \lesssim\left|\psi_{\theta}(n)\right|^{2}+\left|\psi_{\theta}(n+1)\right|^{2} \lesssim n^{\eta}
$$

for all $\theta$ and $n>0$. Then any spectral measure for $h_{V}$ gives no weight to subsets of $A$ of Hausdorff dimension less than $1-\eta$.

We now complete the proof of Theorem 2 by putting these ingredients together.

Proof of Theorem [ We saw above that the absolutely continuous spectrum of $h_{V}$ is essentially supported on $[-2,2]$ and has multiplicity two. Also, by Proposition 3.1. 0 is not an eigenvalue.

The singular part of any spectral measure for $h_{V}$ gives no weight to the set of energies for which all solutions of (6) are bounded (see, e.g., 20, 22, 26]). Thus Proposition 3.2 shows that the singular part must be supported on a set of zero Hausdorff dimension; while Propositions 3.1 and 3.3 together with the previous paragraph imply that it must give no weight to any zero-dimensional subset of $(-2,2)$. Thus it remains only to show that \pm 2 are not eigenvalues. To do so, we mimic the proof of Corollary 4.6 from 6 .

Assume that $E=2$ is an eigenvalue. Then, after possibly changing the value of $V(0)$, we see that $h_{V}^{+}$also has an eigenvalue at 2 , but only finitely many bound states. This contradicts [6, Corollary 4.6 (e)]. The same line of reasoning works when one assumes that $E=-2$ is an eigenvalue.

Proof of Theorem 3 . The proof is analogous to that of Theorem 2. Let us write $\psi_{\theta}$ for the solution of $-\psi^{\prime \prime}(x)+V(x) \psi(x)=E \psi(x)$ that obeys the initial condition $\psi_{\theta}(0)=\sin (\theta), \psi_{\theta}^{\prime}(0)=\cos (\theta)$.

The analogues of the three propositions above can be found in the literature: In place of Proposition 3.1] we can use Corollary 6.5(a) and Propositions 7.4 from [6]. Similarly, Corollary 6.5(a) and Proposition 7.7 from [6] substitute Proposition 3.2. That the continuum analogue of Proposition 3.3 also holds, was noted already in [7.

Finally, showing that zero is not an eigenvalue, can be effected by mimicking the proof of [6] Corollary 6.5(b)].

\section{Perturbations of Operators With Positive Ground States}

In this section we extend the variational technique introduced in [4: we are able to treat continuum Schrödinger operators and also allow more general unperturbed operators, specifically, those with positive ground states. As there is greater novelty in the continuum case, this is what will be presented. Adapting these proofs to the discrete case is a fairly elementary exercise.

The key computation from [4] is the following, whose proof is straightforward.

Lemma 4.1. If $H$ and $V$ are self-adjoint operators and $f$ and $g$ are vectors in the form domains of both operators, then

$$
\begin{aligned}
& \langle f+\varepsilon g|H+V| f+\varepsilon g\rangle+\langle f-\varepsilon g|H-V| f-\varepsilon g\rangle \\
= & 2\langle f|H| f\rangle+4 \varepsilon \operatorname{Re}\langle f|V| g\rangle+2 \varepsilon^{2}\langle g|H| g\rangle .
\end{aligned}
$$


We will also need the following little computational lemma that appears to go back to Jacobi [12] (see also Courant-Hilbert [3] p. 458]):

Lemma 4.2. Let a be an $H^{1}=W^{1,2}$ function of compact support on $\mathbb{R}^{d}$. If $Q$ is locally $L^{1}$ and $\psi \in W_{\mathrm{loc}}^{1,1}$ is a real-valued solution of $-\nabla^{2} \psi+Q \psi=0$ then

$$
\int\|\nabla(a \psi)\|^{2}+Q(a \psi)^{2}=\int\|\nabla a\|^{2} \psi^{2} .
$$

Proof. Integrating by parts and using $-\nabla^{2} \psi+Q \psi=0$,

$$
\int a^{2}\|\nabla \psi\|^{2}=-\int \psi \nabla \cdot\left(a^{2} \nabla \psi\right)=-\int 2 a \psi(\nabla a) \cdot(\nabla \psi)+Q a^{2} \psi^{2}
$$

and consequently

$$
\begin{aligned}
\int\|\nabla(a \psi)\|^{2}+Q(a \psi)^{2} & =\int\|a \nabla \psi+(\nabla a) \psi\|^{2}+Q a^{2} \psi^{2} \\
& =\int a^{2}\|\nabla \psi\|^{2}+2 a \psi(\nabla a) \cdot(\nabla \psi)+\|\nabla a\|^{2} \psi^{2}+Q a^{2} \psi^{2} \\
& =\int\|\nabla a\|^{2} \psi^{2}
\end{aligned}
$$

as promised.

Proof of Theorem 5. Without loss of generality, we assume that the ground state energy of $H_{Q}$ is zero. Let $\psi$ be a bounded positive ground state for $H_{Q}$ and assume that $V \not \equiv 0$. Then there exist $M>0$ and a smooth function $g$, supported in the ball of radius $M$ centered at the origin, such that

$$
\int \psi V g<0
$$

Given $N>M$, define $a$ as follows: in one dimension,

$$
a(x)= \begin{cases}0 & |x| \geq N \\ 1 & |x| \leq M \\ 1-\frac{|x|-M}{N-M} & M<|x|<N\end{cases}
$$

and in two dimensions,

$$
a(x)= \begin{cases}0 & |x| \geq N \\ 1 & |x| \leq M \\ \frac{\log N-\log |x|}{\log N-\log M} & M<|x|<N .\end{cases}
$$

Applying Lemma 4.2 with $f=a \psi$, we obtain

$$
\langle f|H| f\rangle=\int\|\nabla f\|^{2}+Q f^{2}=\int\|\nabla a\|^{2} \psi^{2} \lesssim \int\|\nabla a\|^{2} .
$$


In both one and two dimensions, the right-hand side converges to zero as $N \rightarrow \infty$; in one dimension it is easily seen to be $O\left(N^{-1}\right)$, for two dimensions,

$$
\begin{aligned}
\int\|\nabla a\|^{2} & =\int\|\nabla a\|^{2} r d r d \theta \\
& =2 \pi[\log (N / M)]^{-2} \int_{M}^{N} r^{-1} d r \\
& =2 \pi[\log (N / M)]^{-1} .
\end{aligned}
$$

This shows that $\langle f|H| f\rangle \rightarrow 0$ as $N \rightarrow \infty$.

By (9), we can choose $\varepsilon>0$ small enough so that

$$
4 \varepsilon \operatorname{Re}\langle f|V| g\rangle+2 \varepsilon^{2}\langle g|H| g\rangle=4 \varepsilon \operatorname{Re}\langle\psi|V| g\rangle+2 \varepsilon^{2}\langle g|H| g\rangle<0 .
$$

Therefore, Lemma 4.1] shows that, for $N$ large enough,

$$
\left\langle f+\varepsilon g\left|H_{Q}+V\right| f+\varepsilon g\right\rangle+\left\langle f-\varepsilon g\left|H_{Q}-V\right| f-\varepsilon g\right\rangle<0 .
$$

Thus, at least one of $H_{Q \pm V}$ has spectrum below zero.

The proof shows that we need not have assumed that the ground state is bounded, but merely has sufficiently slow growth at infinity. For example, $|\psi(r, \theta)|=o(\log (r))$ would suffice in two dimensions. In one dimension, however, we can refine this idea and prove the following:

Theorem 7. Suppose that $Q \in L_{\mathrm{loc}}^{2}(\mathbb{R})$ and the operator $H_{Q}$ has a positive ground state whose reciprocal is not square-integrable both at $+\infty$ and at $-\infty$. If $V \in$ $L_{\text {loc }}^{2}(\mathbb{R})$ and both $H_{Q \pm V}$ are bounded below by the ground state energy of $H_{Q}$, then $V \equiv 0$.

Proof. The main idea is to choose $a$ in a manner that is adapted to $\psi$ :

$$
a(x)= \begin{cases}0 & |x| \geq N \\ 1 & |x| \leq M \\ 1-\frac{\int_{M}^{x} \psi(t)^{-2} d t}{\int_{M}^{N} \psi(t)^{-2} d t} & M<x<N \\ 1-\frac{\int_{x}^{-M} \psi(t)^{-2} d t}{\int_{-N}^{-M} \psi(t)^{-2} d t} & -N<x<-M .\end{cases}
$$

This necessitates only one change to the proof, namely, the calculation which shows that $\langle f|H| f\rangle \rightarrow 0$ as $N \rightarrow \infty$.

Note that $a$ is compactly supported and belongs to $H^{1}$; in fact, $a^{\prime} \in L^{\infty}$ (as a distribution). Therefore, we can apply Lemma 4.2 to obtain

$$
\begin{aligned}
\int\left(f^{\prime}\right)^{2}+Q f^{2} d x & =\int\left(a^{\prime}\right)^{2} \psi^{2} d x \\
& =\int_{M}^{N} \frac{d x}{\psi(x)^{2}\left\{\int_{M}^{N} \psi(t)^{-2} d t\right\}^{2}}+\int_{-N}^{-M} \frac{d x}{\psi(x)^{2}\left\{\int_{-N}^{-M} \psi(t)^{-2} d t\right\}^{2}} \\
& =\left\{\int_{M}^{N} \psi(t)^{-2} d t\right\}^{-1}+\left\{\int_{-N}^{-M} \psi(t)^{-2} d t\right\}^{-1} .
\end{aligned}
$$

By assumption, the right-hand side goes to zero as $N \rightarrow \infty$. 


\section{REFERENCES}

[1] S. Agmon, On positive solutions of elliptic equations with periodic coefficients in $\mathbb{R}^{n}$, spectral results and extensions to elliptic operators on Riemannian manifolds, in Differential Equations (Birmingham, Ala., 1983), North-Holland Math. Stud. 92, North-Holland, Amsterdam (1984), 7-17

[2] H. Behncke, Absolute continuity of Hamiltonians with von Neumann Wigner potentials. II., Manuscripta Math. 71 (1991), 163-181

[3] R. Courant and D. Hilbert, Methods of Mathematical Physics. Vol. I., Interscience Publishers, Inc., New York, 1953

[4] D. Damanik, D. Hundertmark, R. Killip, and B. Simon, Variational estimates for discrete Schödinger operators with potentials of indefinite sign, Commun. Math. Phys. 238 (2003), $545-562$

[5] D. Damanik, D. Hundertmark, and B. Simon, Bound states and the Szegö condition for Jacobi matrices and Schrödinger operators, J. Funct. Anal. 205 (2003), 357-379

[6] D. Damanik and R. Killip, Half-line Schrödinger operators with no bound states, to appear in Acta Math.

[7] D. Damanik, R. Killip, and D. Lenz, Uniform spectral properties of one-dimensional quasicrystals. III. $\alpha$-continuity, Commun. Math. Phys. 212 (2000), 191-204

[8] D. Damanik and C. Remling, in preparation

[9] D. J. Gilbert, On subordinacy and analysis of the spectrum of Schrödinger operators with two singular endpoints, Proc. Roy. Soc. Edinburgh Sect. A 112 (1989), 213-229

[10] D. J. Gilbert and D. B. Pearson, On subordinacy and analysis of the spectrum of onedimensional Schrödinger operators, J. Math. Anal. Appl. 128 (1987), 30-56

[11] D. Hundertmark and B. Simon, Lieb-Thirring inequalities for Jacobi matrices, J. Approx. Theory 118 (2002), 106-130

[12] C. Jacobi, Zur Theorie der Variations-Rechnung und der Differential-Gleichungen, J. Reine Angew. Math. 17 (1837), 68-82

[13] S. Jitomirskaya and Y. Last, Power-law subordinacy and singular spectra. I. Half-line operators, Acta Math. 183 (1999), 171-189

[14] R. Killip and B. Simon, Sum rules for Jacobi matrices and their applications to spectral theory, Ann. of Math. 158 (2003), 253-321

[15] W. Kirsch and B. Simon, Corrections to the classical behavior of the number of bound states of Schrödinger operators, Ann. Physics 183 (1988), 122-130

[16] P. B. Nauman, The set of isolated points of increase of the spectral function pertaining to a limit-constant Jacobi matrix (Russian), Izv. Vyš̌. Učebn. Zaved. Matematika 1 (8) (1959), 129-135

[17] F. Peherstorfer and P. Yuditskii, Asymptotics of orthonormal polynomials in the presence of a denumerable set of mass points, Proc. Amer. Math. Soc. 129 (2001), 3213-3220

[18] Y. Pinchover, On positive solutions of second-order elliptic equations, stability results, and classification, Duke Math. J. 57 (1988), 955-980

[19] Y. Pinchover, On criticality and ground states of second order elliptic equations. II, J. Differential Equations 87 (1990), 353-364

[20] R. G. Pinsky, Second order elliptic operators with periodic coefficients: criticality theory, perturbations, and positive harmonic functions, J. Funct. Anal. 129 (1995), 80-107

[21] O. Safronov, Multi-dimensional Schrödinger operators with no negative spectrum, preprint (mp-arc/04-273)

[22] B. Simon, Bounded eigenfunctions and absolutely continuous spectra for one-dimensional Schrödinger operators, Proc. Amer. Math. Soc. 124 (1996), 3361-3369

[23] B. Simon, The Golinskii-Ibragimov method and a theorem of Damanik and Killip, Int. Math. Res. Not. 2003, 1973-1986

[24] B. Simon, Schrödinger semigroups, Bull. Amer. Math. Soc. 7 (1982), 447-526

[25] B. Simon and A. Zlatoš, Sum rules and the Szegő condition for orthogonal polynomials on the real line, Commun. Math. Phys. 242 (2003), 393-423

[26] G. Stolz, Bounded solutions and absolute continuity of Sturm-Liouville operators, J. Math. Anal. Appl. 169 (1992), 210-228

[27] H. Tamura, The Efimov effect of three-body Schrödinger operators, J. Funct. Anal. 95 (1991), 433-459 
Mathematics 253-37, California Institute of Technology, Pasadena, CA 91125, USA

E-mail address: damanik@caltech.edu

Department of Mathematics, University of California, Los Angeles, CA 90055, USA

E-mail address: killip@math.ucla.edu

Mathematics 253-37, California Institute of Technology, Pasadena, CA 91125, USA

E-mail address: bsimon@caltech.edu 